\title{
SUCCESSFUL MANAGEMENT OF SEVERE EXERTIONAL HEAT STROKE COMPLICATED BY VENTRICULAR FIBRILLATION IN 19 YEARS OLD MALE
}

\author{
Tomasz Klosiewicz ${ }^{\circledR}$, Natalia Ciepluch ${ }^{\circ}$, Roland Podlewski®
}

Department of Medical Rescue, Poznan University of Medical Sciences, Poznan, Poland

\begin{abstract}
Heatstroke is a heat-related disease that is highly fatal. However, it is significant that if symptoms are quickly recognized, the outcome remains satisfactory. In this paper, the authors present the case of a 19-year-old physical worker in whom heatstroke was complicated by cardiac arrest. The proper action and full diagnosis of the underlying cause of cardiac arrest allowed for a full recovery. Attention was also drawn to the lack of appropriate equipment for active cooling and potential use of automatic chest compressions devices.
\end{abstract}

KEY WORDS: exertional heat stroke, sudden cardiac arrest, prehospital care

Disaster Emerg Med J 2020; 5(4)

\section{INTRODUCTION}

Heatstroke is one of the forms of overheating associated disease. Early recognition of symptoms and withdrawal of exposure to heat, as well as activation of appropriate treatment, enable to avoid complications [1]. Heatstroke is a life-threatening condition with a high risk of death. In France, mortality rates have been reported to be 58 and $71 \%$ for 28 -day and 2 -year, respectively. Moreover, the authors of this research highlighted that by the 2050s, heat stroke-related deaths are expected to rise by 2.5 times. Also, dramatic alteration in functional status was recorded in most surviving patients. If the symptoms are diagnosed early enough, and the appropriate treatment is initiated, heatstroke can be prevented [2].

Sudden Cardiac Arrest (SCA) accounts for approximately $1 \%$ of all emergency ambulance responses [3]. The European Resuscitation Council (ERC) defined reversible causes of cardiac arrest. These include, among others, hypovolaemia, electrolyte disorders, and coronary embolism. During cardiopulmonary resuscitation (CPR), all of them should be considered and treated if necessary. The survival of out-of-hospital-hospital SCA remains unsatisfactory [4]. Providing CPR in a two-rescuer team is a big challenge. It has been shown that such a team cannot perform all activities according to the recommended algorithm [5].

In this paper, the authors reported a case of a young man in whom heat stroke was complicated by SCA. The authors aimed to highlight the problem of overheating associated disease and indicate potential difficulties in patient care.

\section{CASE REPORT}

On a hot summer day (ambient temperature $37^{\circ} \mathrm{C}$ ) at 18:13, the Medical Rescue Team, consisting of two paramedics, responded to SCA in a 19 years-old male. The patient was working on building a stable. According to the witnesses of the incident, the cause of the SCA was probably an electric shock.

The paramedics arrived at the scene after about 7 minutes. During the initial assessment, the para- 
medics paid attention to the electric wires lying near the patient. From bystanders' reports, at the time of the event, the victim was repairing the hydrophor - hence the electric shock (230V) was highly suspected. The owner of the property could not disconnect the power source - he did not know where the main switch was located. The paramedics confirmed the cardiac arrest and immediately took over the CPR. The first rhythm was ventricular fibrillation (VF). Therefore, 200J energy defibrillation was performed. After the cardiac arrest had been confirmed, a physician-staffed helicopter emergency medical service (HEMS) was dispatched. Due to the lack of family at the site, the detailed medical history was impossible to be collected. The witnesses of the event presented inconsistent accounts. Due to difficulties in determining the course of events and the impossibility of excluding the participation of third parties, the Police was called.

The airway was secured with an oropharyngeal tube, bag-valve-mask ventilation was applied, and a peripheral G18 intravenous access was established. After 2 minutes of resuscitation, the monitor presented ventricular tachycardia (VT) with QRS frequency exceeding 180/min. The presence of pulse on the carotid artery and no pulse on peripheral arteries were confirmed. The patient was breathing spontaneously at a rate of 20/min. Oxygen saturation was $92 \%$. The skin was pale, sweaty, warm, without visible external injuries suggesting trauma or electric shock. Systolic blood pressure $70 \mathrm{mmHg}$. Pupils narrow, equal, and adequately responding to light. Capillary glucose level was $194 \mathrm{mg} / \mathrm{dl}$. No obvious injuries revealed in the head-to-toe examination.

The cooling treatment was implemented. The patient was transferred to an air-conditioned ambulance. The body was covered in hydrogel dressings. Oxygen supplementation was initiated. Normal sinus rhythm was restored with $150 \mathrm{mg}$ of amiodarone. Hypovolaemia was treated with repetitive boluses of crystalloids. Due to agitation, $5 \mathrm{mg}$ of midazolam was administered.

In the meantime, the paramedics were informed by the witnesses of the incident that the patient had been working in full sun for 7 hours without headgear for laying paving stones. He also did not take enough fluids. This information, together with the lack of evidence confirming the electric shock, made the victim of hyperthermia suspected. This hypothesis was confirmed by the value of the measured body temperature in the tympanic membrane. The thermometer indicated $41.2^{\circ} \mathrm{C}$.

HEMS doctor decided to perform endotracheal intubation in the rapid sequence intubation protocol. In the ultrasound performed on the e-FAST protocol, the presence of free fluid in the abdomen and pneumothorax were excluded. A Foley catheter was inserted, and $20 \mathrm{mg}$ of furosemide was administered. The patient was transported to the emergency department by HEMS.

The patient was admitted to the emergency department with full consciousness. Echocardiography and CT scan were performed. These tests did not show any abnormalities. Myocardial ischemia requiring urgent coronary angiography was also not found. Laboratory tests revealed the following abnormalities: leucocytes: $25.2 * 10 \wedge 3 / \mu \mathrm{L}$, ALT: 617 U/L, AST: 448 U/L, Creatinine: $127 \mu \mathrm{mol} / \mathrm{L}$, Chloride: 109 mmol/L, cTnl: 182ng/L, CKI: 355 IU/L. The patient was transferred to the Intensive Care Unit and discharge after 11 days with full recovery and no neurological disabilities.

During the whole event, the 12-lead ECG was performed several times. At first, VT was present. After the sinus rhythm was restored, the flattening and then reversion of T-waves were revealed.

\section{DISCUSSION}

Heatstroke is the last expression of the body's reaction to overheating. It occurs when the physiological mechanisms are not sufficient to release the accumulated excess heat. The phases preceding heatstroke are: overheating with heat cramps and heat exhaustion. A distinction is made between: Exercise Heat Stroke (EHS) and Non-Exercise Heat Stroke (NEHS), also called the Classical Heat Stroke. By definition, EHS is a hyperthermic condition that occurs in individuals performing intense exercise, typically in warm environments. EHS is characterized by severe hyperthermia $\left(>40.5^{\circ} \mathrm{C}\right.$ ) and organ insufficiency, which typically manifests as central nervous system (CNS) dysfunction. It occurs most often in healthy people, young people, working or exercising in high-temperature conditions [6]. In contrast, NCHS occurs mainly in the elderly and infants. In these age groups, the physiological mechanisms of thermoregulation are compromised. However, it should be noted that even in cooler conditions, strenuous physical activity by itself may result in an individual succumbing to EHS [7]. 
Physiological body temperature is maintained at approximately $37.5^{\circ} \mathrm{C}$ by the hypothalamus. The mechanisms related to heat exchange are radiation, convection, conduction, and vaporization. The effect of temperature increase is the dilation of blood vessels in the skin. This process supports the abovementioned thermoregulatory mechanisms. The skin may be red and hot. In the first phase, when the thermoregulatory mechanisms are effective, abundant sweating is observed. When these mechanisms fail, the skin becomes dry. Lack of sweat is one of the symptoms that distinguish heat stroke from heat cramps and heat exhaustion. Cutaneous vasodilation causes the blood to move to the peripheral parts of the body. This, in turn, leads to relative hypovolemia. Syncope may be the first symptom observed by witnesses. As a result, reduced organ perfusion causes progressive multiorgan failure. Water and electrolytes are lost as a result of perspiration. Tachycardia and hypotension may be present. Muscle cramps can be the presentation of dyselectrolytemia. In patients with impaired peripheral perfusion, the skin may be pale and sweaty. At this point, it is worth mentioning that the temperature tolerance is different for different people. The body's response depends, among other things, on the overall performance of the cardiovascular system. It is therefore essential not to make a decision solely based on temperature measurement, but after a thorough examination of the patient $[6,8,9]$. Direct cytotoxic effects of heat followed by the inflammatory and coagulation responses, injure the vascular endothelium. This might be clinically manifest as DIC [1]. In NEHS, there is a high prevalence of atrial arrhythmias, low-voltage $p$ waves, low limb lead QRS voltage, QTc prolongation, right bundle branch block and repolarisation abnormalities [10].

The ERC recommends taking temperature measurements in the oesophagus, rectum, bladder, or tympanic membrane [11]. Of the methods available for emergency teams, only the latter is possible. In the case of hypothermia, accurate measurement is much more critical. The patient's classification into the appropriate therapeutic class of hypothermia depends on the exact body temperaturę [12]. In the case of overheat illnesses, the combination of history and symptoms with high body temperature is crucial. Incidents, where the death occurred in patients with both lower and extremely high body temperatures, were described.
The purpose of cooling treatment is to reduce the time when the patient is hyperthermic. The notable increase in organ damage and mortality occurs after 30 minutes of hyperthermia. Thus, rapid cooling must be provided in 30 minutes from the time of collapse [13]. The recommended rate of cooling is at least $0.15^{\circ} \mathrm{C}$ per minute. Cooling should be finished when the body temperature reaches $38.6^{\circ} \mathrm{C}$ to minimize the risk of severe hypothermia. The goal is to create conditions where the energy accumulated in the body could be transferred to an external medium. It is most beneficial to use all the mechanisms of heat exchange. Coldwater immersion is considered to be the standard gold treatment for EHS [1]. Unfortunately, this method is not available in pre-hospital care. In an ambulance, low ambient temperatures, the use of wet or hydrogel dressings can be helpful. If possible, a cooling airflow should be directed towards the patient. In the presented case, the paramedics used small hydrogel dressings. Such dressings are also available as a blanket. It is also necessary to correct hypovolemia and electrolyte disruptions. At present, in the Polish Medical Rescue System, there is no equipment dedicated to the treatment of heatstroke.

Ventricular fibrillation (VF) may occur as a result of acute myocardial ischemia, electrolyte disorders or electric shock. In a young person also cardiomyopathy should be considered. In this case, the patient was young, thus cardiovascular diseases are unlikely. However, the initial assessment of the situation indicated the possibility of electrocution. Young age, VF, CPR has undertaken by witnesses of the event and short duration of SCA are positive prognostic factors $[14,15]$.

In the case of CPR extending beyond 15 minutes, the patient should also be considered for extracorporeal CPR (ECPR). The use of extracorporeal oxygenation membrane (ECMO) therapy increases the patient's chances of survival. In the case of irreversible cardiac arrest, however, it allows sustaining organ perfusion for transplantation. Puslecki et al. performer high fidelity medical simulations, proving the possibility of combine ECMO and pre-hospital care in Poland [16-18].

Providing CPR under the conditions on the scene can be challenging. Chest compressions are exhausting for the rescuer. There are currently no clear recommendations for the routine use of automatic chest compression devices (ACCD). A positive effect of these devices on the long-term survival of an SCA incident has not been defined. However, ACCDs pro- 
vide high-quality compressions, especially in a team of only two people [19]. Under such environmental conditions, the use of ACCD may be necessary to maintain high-quality chest compressions.

The ambulance team noted difficulties in attaching ECG leads to the patient - despite an attempt to dry the victim's sweaty, damp chest, the electrodes did not adhere properly to the patient's body. A similar situation can occur for any patient in shock. Alternative fixtures such as pilers and bulb suckers are a good alternative. They do not require sticking but must be disinfected after each use. Currently rarely used in ambulances.

In the presented case, attention is drawn to the lack of ability of construction workers to disconnect the power source. Additionally, the lack of headgear, an insufficient supply of fluids, and the lack of breaks in work shows low awareness of workers in terms of occupational health and safety.

\section{CONCLUSIONS}

The analysis of the presented case suggests that Medical Rescue Teams are not properly prepared to treat EHS patients. Attention should be paid to providing appropriate resources for quick and effective body cooling - especially during the summer. It may also be useful to create a prehospital EHS treatment procedure. High ambient temperatures should be considered as a potential indication for the use of ACCD for safety reasons.

Conflict of interests: The authors do not declare.

\section{REFERENCES}

1. Belval LN, Casa DJ, Adams WM, et al. Consensus Statement- Prehospital Care of Exertional Heat Stroke. Prehosp Emerg Care. 2018; 22(3): 392-397, doi: 10.1080/10903127.2017.1392666, indexed in Pubmed: 29336710.

2. Argaud L, Ferry $T$, Le $Q H$, et al. Short- and long-term outcomes of heatstroke following the 2003 heat wave in Lyon, France. Arch Intern Med. 2007; 167(20): 2177-2183, doi: 10.1001/archinte.167.20. ioi70147, indexed in Pubmed: 17698677.

3. Kłosiewicz T, Skitek-Adamczak I, Zieliński M. Emergency medical system response time does not affect incidence of return of spontaneous circulation after prehospital resuscitation in one million central European agglomeration residents. Kardiol Pol. 2017; 75(3): 240-246, doi: 10.5603/KP.a2016.0181, indexed in Pubmed: 27995600.

4. Soar J, Nolan J, Böttiger B, et al. European Resuscitation Council Guidelines for Resuscitation 2015. Resuscitation. 2015; 95: 100-147, doi: 10.1016/j.resuscitation.2015.07.016.
5. Dabrowski M, Sip M, Dabrowska A, et al. It is impossible to follow the ERC algorithm in a two-paramedics ambulance team. Resuscitation. 2017; 118: e43, doi: 10.1016/j.resuscitation.2017.08.108.

6. Bouchama A, Knochel JP. Heat stroke. N Engl J Med. 2002; 346(25): 19781988, doi: 10.1056/NEJMra011089, indexed in Pubmed: 12075060.

7. Roberts WO. A 12-yr profile of medical injury and illness for the Twin Cities Marathon. Med Sci Sports Exerc. 2000; 32(9): 1549-1555, doi: 10.1097/00005768-200009000-00004, indexed in Pubmed: 10994903.

8. Miyake M. Pathophysiology of Heat Illness: Thermoregulation, risk factors, and indicators of aggravation. Japan Med Assoc J. 2013; 56: 167-173.

9. Hifumi T, Kondo Y, Shimizu K, et al. Heat stroke. J Intensive Care. 2018; 6: 30, doi: 10.1186/s40560-018-0298-4, indexed in Pubmed: 29850022.

10. Paul $A$, Alex R, Jacob JR, et al. Effects of heat stroke on surface ECG: a study on clinical outcomes. Heart Asia. 2019; 11(2): e011221, doi: 10.1136/heartasia-2019-011221, indexed in Pubmed: 31297165.

11. Soar J, Perkins GD, Abbas $G$, et al. European Resuscitation Council Guidelines for Resuscitation 2010 Section 8. Cardiac arrest in special circumstances: Electrolyte abnormalities, poisoning, drowning, accidental hypothermia, hyperthermia, asthma, anaphylaxis, cardiac surgery, trauma, pregnancy, electrocution. Resuscitation. 2010; 81(10): 1400-1433, doi: 10.1016/j.resuscitation.2010.08.015, indexed in Pubmed: 20956045.

12. Kłosiewicz T, Zalewski R. Hipotermia pourazowa jako wyzwanie dla personelu zespołów ratownictwa medycznego. Pomeranian Journal of Life Sciences. 2018; 64(4), doi: 10.21164/pomjlifesci.484.

13. Heled $Y$, Rav-Acha $M$, Shani $Y$, et al. The "golden hour" for heatstroke treatment. Mil Med. 2004; 169(3): 184-186, doi: 10.7205/ milmed.169.3.184, indexed in Pubmed: 15080235.

14. Rajan S, Folke F, Kragholm K, et al. Prolonged cardiopulmonary resuscitation and outcomes after out-of-hospital cardiac arrest. Resuscitation. 2016; 105: 45-51, doi: 10.1016/j.resuscitation.2016.05.004, indexed in Pubmed: 27224447.

15. Ong ME, Perkins GD, Cariou A. Out-of-hospital cardiac arrest: prehospital management. Lancet. 2018; 391(10124): 980-988, doi: 10.1016/S0140-6736(18)30316-7, indexed in Pubmed: 29536862.

16. Michels $G$, Wengenmayer $T$, Hagl C, et al. Recommendations for extracorporeal cardiopulmonary resuscitation (eCPR): consensus statement of DGIIN, DGK, DGTHG, DGfK, DGNI, DGAI, DIVI and GRC. Clin Res Cardiol. 2019; 108(5): 455-464, doi: 10.1007/s00392-018-1366-4, indexed in Pubmed: 30361819.

17. Kłosiewicz T, Puślecki M, Zieliński M, etal. The role of extracorporeal membrane oxygenation in patients after irreversible cardiac arrest as potential organ donors. Kardiochir Torakochirurgia Pol. 2017; 14(4): 253-257, doi: 10.5114/kitp.2017.72230, indexed in Pubmed: 29354178.

18. Puślecki $M$, Ligowski $M$, Stefaniak $S$, et al. Using simulation to create a unique regional ECMO program for the Greater Poland region. Qatar Medical Journal. 2017; 2017(1): 79, doi: 10.5339/qmj.2017.swacelso.79.

19. Kłosiewicz T, Puślecki M, Zalewski $R$, et al. Analysis of the quality of chest compressions during resuscitation in an understaffed team — randomised crossover manikin study. Disaster and Emergency Medicine Journal. 2020; 5(1): 24-29, doi: 10.5603/demj.a2020.0008. 